\title{
22. Spotlight: Welche Folgen hat das EuGH- Urteil zur rechtlichen Einordnung von Mutagenese-Organismen?
}

\subsection{EuGH-Urteil vom 25. Juli 2018}

Am 25. Juli 2018 hat der Europäische Gerichtshof (EuGH) in der Rechtssache C-528/16, Confédération paysanne u. a., ein wegweisendes Urteil für die Regulierung genomeditierter Organismen erlassen. Explizit entschieden - in Gestalt von Antworten auf Fragen des französischen Conseil d'État - hat er aber eigentlich allein, dass (1) „mit Verfahren/Methoden der Mutagenese gewonnene [...] Organismen GVO [...] darstellen“ und (2) „nur die mit Verfahren/Methoden der Mutagenese, die herkömmlich bei einer Reihe von Anwendungen angewandt wurden und seit langem als sicher gelten, gewonnenen Organismen [...] ausgeschlossen“, ${ }^{1} \mathrm{~d}$. h. von der Regulierung durch die Richtlinie 2001/18/EG ${ }^{2}$ ausgenommen sind. ${ }^{3}$ Auch wenn in diesen, die

1 EuGH, C-528/16, Confédération paysanne u. a., ECLI:EU:C:2018:583: 54.

2 Richtlinie 2001/18/EG des Europäischen Parlaments und des Rates vom 12. März 2001 über die absichtliche Freisetzung genetisch veränderter Organismen in die Umwelt und zur Aufhebung der Richtlinie 90/220/EWG des Rates. In: ABl. EU L 106, 17.04.2001: 1-39.

3 Die herkömmlichen, seit vielen Jahrzehnten eingesetzten Verfahren der Mutagenese verwenden Chemikalien oder ionisierende Bestrahlung, um mit deren Hilfe zufällige Mutationen in Pflanzen und darüber verbesserte oder neuartige Pflanzeneigenschaften zu erzeugen. Durch zahlreiche weitere Schritte der Selektion und Rückkreuzung lassen sich auf diesem Wege neue marktfähige Zier- oder Nutzpflanzensorten züchten. Das EuGH-Urteil stuft auch solche, aus lange etablierten Methoden der Mutagenese hervorgegangenen Pflanzen als gentechnisch veränderte Organismen (GVO) ein, nimmt sie jedoch sogleich wieder von der Regulierung aus, weil sie bzw. ihre Verwendung seit langem als sicher gelten. Mit den modernen Techniken der Genomeditierung können nunmehr gezielte Mutationen an vorabdefinierten Stellen im Genom von Pflanzen erzeugt werden. Strittig war bis zum Urteil des EuGH vom 25. Juli 2018, ob aus solchen hochinnovativen Mutageneseverfahren hervorgegangene Pflanzen GVO sind und, bejahendenfalls, ob sie wie mittels herkömmlicher Mutagenese gezüchtete Pflanzen sogleich wieder aus dem GVO-Regelwerk herausfallen. 
Urteilsformel $^{4}$ bildenden Antworten die Genomeditierung ${ }^{5}$ nicht expressis verbis genannt wird, ist die Schlussfolgerung gleichwohl zutreffend, dass nunmehr alle genomeditierten Organismen ausnahmslos von der Richtlinie 2001/18/EG regulierte genetisch veränderte Organismen (GVO) im Rechtssinne sind (z. B. Faltus, 2018: 531; Kahrmann/Leggewie, 2018: 761; Seitz, 2018: 763; Spranger, 2018: 2929; Voigt, 2018: 654/657 f./662; Beck, 2019: 246; Dederer, 2019: 236; Leopoldina et al., 2019: 3; Voigt/Münichsdorfer, 2019: 147 ff.; Wasmer, 2019: 3; Purnhagen/Wesseler, 2020: 2).

Verständlich wird diese Reichweite der oben zitierten Antworten des EuGH nur mit Blick auf die Urteilsbegründung und die prozessuale Situation, ${ }^{6}$ in welcher der Gerichtshof sein Urteil gefällt hat. ${ }^{7}$ Konkret ging es um ein Vorlageverfahren eines nationalen Gerichts, welches dem EuGH Fragen zur Auslegung des Unionsrechts vorgelegt hatte. In einem solchen Verfahren zur Vorabentscheidung von Interpretationsfragen (vgl. Art. 267 Abs. 1 lit. b AEUV) ${ }^{8}$ gibt der EuGH dem vorlegenden nationalen Gericht ,alle Kriterien für die Auslegung des Unionsrechts an die Hand, die das Gericht in die Lage versetzen, die [Auslegung und Anwendung des nationalen Rechts im Lichte] der Unionsregelung zu beurteilen“. 9 Wegen der:

„Notwendigkeit, zu einer dem nationalen Gericht nützlichen Auslegung des [Unions]rechts zu gelangen, [ist] es [aber] erforderlich [...], daß [das nationale] Gericht den tatsächlichen und rechtlichen Rahmen, in den sich die von ihm gestellten

4 Ein Urteil besteht im Wesentlichen, vereinfacht und allgemein dargestellt, aus drei Elementen: der „Urteilsformel“ (= Urteil „im engeren Sinn“), dem „Tatbestand“ (= dem Urteil zugrunde liegender Sachverhalt einschließlich Verfahrensgang) und den „Gründen“ (= die Urteilsfindung leitende Begründung). In sog. „materieller“ Rechtskraft erwächst grundsätzlich nur die Urteilsformel, d. h. nach Eintritt der ,formellen“ Rechtskraft (= Unanfechtbarkeit) des Urteils bindet die am Rechtsstreit Beteiligten nur das, was in dieser Urteilsformel vom Gericht inhaltlich entschieden worden ist. Die Reichweite dessen, was das Gericht in der Urteilsformel inhaltlich entschieden hat und Bindungswirkung zwischen den Streitbeteiligten entfaltet, ist aber ggf. durch Auslegung anhand der Gründe und u. U. mit Blick auf den Tatbestand zu ermitteln.

5 Dabei werden unter Verfahren der Genomeditierung die oligonukleotid-gelenkte Mutagenese („oligonucleotide-directed mutagenesis“ - ODM) und die mit ortsspezifischen Nukleasen („site-directed nucleases“ - SDN) arbeitenden Methoden der genetischen Veränderung von Organismen verstanden. Siehe nur Gürtler et al., 2019: $7 \mathrm{ff}$.

6 Also mit Blick auf „Gründe“ und „Tatbestand“ im oben in Fußnote 4 genannten Sinn.

7 Siehe oben in Fußnote 4.

8 Vertrag über die Arbeitsweise der Europäischen Union. In: ABl. EU C 202, 07.06.2016: 47-199.

9 EuGH, C-292/92, Hünermund u. a./Landesapothekerkammer Baden-Württemberg, ECLI:EU:C:1993:932: 8. 
Fragen einfügen, festlegt oder zumindest die tatsächlichen Annahmen erläutert, auf denen diese Fragen beruhen“. ${ }^{10}$

In der Folge hat sich der EuGH an die ihm vom mitgliedstaatlichen Gericht gestellten Fragen ebenso zu halten wie an die jenen Fragen vom betreffenden Gericht zugrunde gelegte Darstellung der Fakten des auf nationaler Ebene zu entscheidenden Sachverhalts.

Wie deshalb die Antworten des EuGH in der Rechtssache C-528/16 zu verstehen sind, ist in Ansehung der vom vorlegenden Conseil d'État gestellten Auslegungsfragen und der zu ihrer Erläuterung vorgetragenen Fakten zu beurteilen. Nach Auffassung des Conseil d'État ${ }^{11}$ differenziere die Richtlinie 2001/18/EG zwischen sog. „Transgenese“, durch welche ein oder mehrere neue Gene verwandter oder nicht verwandter Arten in das Genom integriert würden, und zufälliger Mutagenese, welche zufällige Mutationen innerhalb einer DNA-Sequenz vermittels chemischer oder physikalischer Agenzien erzeuge und in vivo, d. h. an ganzen Pflanzen oder deren Teilen, angewandt werde. Während die „Transgenese“ den Verpflichtungen der Richtlinie unterworfen worden sei, sei die Mutagenese von eben jenen ausgenommen worden. Erst nach Erlass der Richtlinie am 12. März 2001 seien weitere Verfahren der Mutagenese entwickelt worden, nämlich die zufällige Mutagenese in vitro an pflanzlichen Zellen sowie die neuartige, gerichtete, Genomeditierung genannte Mutagenese, welche mittels Gentechnik präzise Mutationen in einem Gen ohne Einfügung eines Fremdgens hervorrufe. Hierzu gehörten namentlich ODM und die Mutagenese mittels SDN (zum wissenschaftlichen Sachstand der Grünen Gentechnik siehe Clemens, Kap. 7). ${ }^{12}$

Vor dem Hintergrund dieser Fakten wollte der Conseil d'État wissen, ob (1) „vor allem die neuen Verfahren der gezielten Mutagenese unter Einsatz gentechnischer Verfahren", ${ }^{13}$ also offenbar die von ihm in Bezug genommenen ODM- und SDN-Verfahren, solche Verfahren sind, die GVO im Rechtssinne der Richtlinie 2001/18/EG erzeugen, und, bejahendenfalls, ob (2) ausschließlich solche GVO aus dem Anwendungsbereich der Richtlinie herausfallen, die „mit schon vor Erlass der Richtlinie bestehenden konventionellen Methoden der Zufallsmutagense durch ionisierende Strahlung oder chemische Mutagene erzeugt wurden", ${ }^{14}$ mithin nicht mit solchen Mutageneseverfahren,

10 EuGH, C-320/90, C-321/90 und C-322/90, Telemarsicabruzzo u. a. / Circostel u. a., ECLI:EU:C:1993:26: 6.

11 Zum Folgenden Conseil d'État, $3^{\mathrm{e}}$ et $8^{\mathrm{e}}$ ch., 3 oct. 2016, n³88649, Confédération Paysanne, ECLI:FR:CE CHR:2016:388649.20161003: 23.

12 Zu ODM- und SDN-Verfahren oben in Fußnote 5.

13 EuGH, C-528/16, Confédération paysanne u. a., ECLI:EU:C:2018:583: 25.

14 EuGH, C-528/16, Confédération paysanne u. a., ECLI:EU:C:2018:583: 25. 
die wie die bezeichneten Genomeditierungsverfahren (ODM, SDN) $)^{15}$ erst nach dem Beschluss der Richtlinie 2001/18/EG am 12. März 2001 entwickelt wurden.

\subsection{Folgen des EuGH-Urteils}

\subsubsection{Folgen für die Einordnung genomeditierter Organismen}

Wenn der EuGH nun mit Blick auf die erste der beiden Fragen ohne Unterscheidung nach alten und neuen Verfahren der Mutagenese antwortet, dass „mit Verfahren/Methoden der Mutagenese gewonnene [...] Organismen GVO [...] darstellen“" ${ }^{16}$ dann sind nach alledem auch genomeditierte Organismen GVO im Sinne der Richtlinie 2001/18/ EG. Nicht gefolgt werden kann daher der neuerdings vertretenen These, das EuGHUrteil schließe nicht aus, dass aus bestimmten Verfahren der Genomeditierung hervorgegangene Mutagenese-Organismen keine GVO sein könnten, weil sie schon nicht die Kriterien der GVO-Definition erfüllten (van der Meer et al., 2021: 11 f.). Diese Auffassung übersieht, dass der EuGH explizit die GVO-Definition ausgelegt und genau dabei keinerlei Differenzierung nach der Art der Mutagenese-Technik vorgenommen hat, ${ }^{17}$ obwohl sich eine solche Differenzierung eigentlich nicht nur nach dem Vorlagebeschluss des Conseil d'État, sondern auch deshalb aufgedrängt hätte, weil der Generalanwalt in seinen Schlussanträgen eine Differenzierung für möglich gehalten hatte dergestalt, dass es ,durch Mutagenese gewonnene Organismen geben [kann], die keine GVO [...] sind“. ${ }^{18}$ Derartige Ansätze hat der EuGH mit beredtem Schweigen schlicht übergangen.

Wenn der EuGH mit Blick auf die zweite der beiden Fragen ferner antwortet, dass „nur die mit Verfahren/Methoden der Mutagenese, die herkömmlich bei einer Reihe von Anwendungen angewandt wurden und seit langem als sicher gelten, gewonnenen Organismen [...] ausgeschlossen sind“",19 dann gehören jedenfalls genomeditierte Organismen eindeutig nicht zu den vom Anwendungsbereich der Richtlinie 2001/18/ EG ausgenommenen GVO. Zwar hat der EuGH weder definiert, unter welchen Voraussetzungen von einer „Reihe von Anwendungen“, noch ab wann von einer „seit langem

15 Zur Einordnung als Verfahren der Genomeditierung oben in Fußnote 5 und Conseil d'État, $3^{\mathrm{e}}$ et $8^{\mathrm{e}}$ ch., 3 oct. 2016, n³88649, Confédération Paysanne, ECLI:FR:CECHR:2016:388649.20161003: 23.

16 EuGH, C-528/16, Confédération paysanne u. a., ECLI:EU:C:2018:583: 54.

17 EuGH, C-528/16, Confédération paysanne u. a., ECLI:EU:C:2018:583: 27-38.

18 Generalanwalt Bobek, Schlussanträge, Rechtssache C-528/16, Confédération paysanne u. a.: 66.

19 EuGH, C-528/16, Confédération paysanne u. a., ECLI:EU:C:2018:583: 54. 
als sicher gelten[den]" Anwendung auszugehen ist. ${ }^{20}$ Aber er hat auf die ihm gestellte Frage des Conseil d'État geantwortet, der ausdrücklich wissen wollte, ob nur solche GVO, die „mit schon vor Erlass der Richtlinie bestehenden konventionellen Methoden der Zufallsmutagenese durch ionisierende Strahlung oder chemische Mutagene erzeugt wurden“, oder ob wirklich ,alle durch Mutagenese gewonnenen genetisch veränderten Organismen" vom Anwendungsbereich der Richtlinie ausgenommen sind. ${ }^{21}$ Der EuGH hat diesbezüglich aber, jetzt eindeutige Differenzierungen vornehmend, in seinen Urteilsgründen expressis verbis festgehalten, die Richtlinie könne nicht so ausgelegt werden, dass sie „mit neuen Verfahren/Methoden der Mutagenese, die seit dem Erlass der Richtlinie entstanden sind oder sich hauptsächlich entwickelt haben, gewonnene Organismen von ihrem Anwendungsbereich ausschließt“".22 Damit bezog er sich auf diejenigen „Verfahren/Methoden der gezielten Mutagenese [...], die mit dem Einsatz von Gentechnik verbunden sind, die seit dem Erlass der Richtlinie 2001/18 entstanden sind oder sich hauptsächlich entwickelt haben“. ${ }^{23}$ Das seien diejenigen Verfahren, über welche „das vorlegende Gericht [...] befinden muss“. ${ }^{24}$ Diese Verfahren hat der EuGH wiederum bei seiner Schilderung des Ausgangsverfahrens unter Bezugnahme auf die Ausführungen des vorlegenden französischen Gerichts als „Verfahren/Methoden der gezielten Mutagenese, bei denen neue gentechnische Verfahren wie die Mutagenese mithilfe von Oligonukleotiden oder die Mutagenese mithilfe zielgerichteter Nuklease zur Anwendung kämen“, identifiziert. ${ }^{25}$

\subsubsection{Folgen für „Freisetzung“ und „Inverkehrbringen“}

Dass genomeditierte Organismen von der Richtlinie 2001/18/EG ausnahmslos erfasste GVO sind, gilt zunächst nur für die Richtlinie selbst, welche den Bereich der (experi-

20 Darin liegt ein wesentliches Problem des Urteils, weil deshalb keinesfalls gesichert ist, dass jedwede als „konventionell“ betitelte Mutagenesetechnik zum Ausschluss damit erzeugter Organismen von der GVO-Regulierung führt.

21 EuGH, C-528/16, Confédération paysanne u. a., ECLI:EU:C:2018:583: 25.

22 EuGH, C-528/16, Confédération paysanne u. a., ECLI:EU:C:2018:583: 51.

23 EuGH, C-528/16, Confédération paysanne u. a., ECLI:EU:C:2018:583: 47.

24 EuGH, C-528/16, Confédération paysanne u. a., ECLI:EU:C:2018:583: 47.

25 EuGH, C-528/16, Confédération paysanne u. a., ECLI:EU:C:2018:583: 23. Unklar geblieben ist der EuGH dagegen in der Frage, ob auch die Verfahren der chemisch oder physikalisch induzierten Zufallsmutagenese in vitro an pflanzlichen Zellen zu GVO führen, die gleichwie genomeditierte Organismen nicht von der Richtlinie 2001/18/EG ausgenommen sind. Aufgrund seiner eigenen Ausführungen hat der Conseil d'État diese Folgerungen aus dem EuGH-Urteil aber konsequenterweise gezogen: Conseil d'État, 7 février 2020, Organismes obtenus par mutagenèse: 6. Hierzu ferner Dederer, 2019: 244. 
mentellen) Freisetzung und des (kommerziellen) Inverkehrbringens von allen Arten von GVO abdeckt. Für den Bereich des Inverkehrbringens, d. h. der Vermarktung, gelten daneben aber auch speziellere, produktspezifische Regeln, nämlich für Saat- und Pflanzgut, Lebens- und Futtermittel, Pflanzenschutz- sowie Arzneimittel. Indes definieren jene unionsrechtlichen Regelwerke ihren jeweiligen Anwendungsbereich unter Rekurs auf den Anwendungsbereich der Richtlinie 2001/18/EG. Entsprechendes gilt für die alle Arten von GVO betreffenden Regeln über die Kennzeichnung und Rückverfolgbarkeit sowie die Ausfuhr aus der EU (Dederer, 2019: 243; Dederer, 2020: 46-47). Infolgedessen erstreckt sich der gesamte unionsrechtlich geregelte Bereich der Freisetzung und des Inverkehrbringens von GVO uneingeschränkt auf alle genomeditierten Organismen. ${ }^{26}$

Daraus erwachsen zahlreiche praktische Probleme des Normvollzugs (Leopoldina et al., 2019: 19-21; Dederer, 2020: 47-50; siehe van den Daele/Broer, Kap. 21). Feldversuche mit und jeder kommerzielle Anbau von genomeditierten Pflanzen müssen im öffentlich einsehbaren Standortregister verzeichnet werden, weshalb aufgrund historischer Erfahrung ausnahmslos mit Feldzerstörungen zu rechnen ist. Die Vermarktung genomeditierter Organismen bedarf der Genehmigung, die gleichfalls, nach Erfahrungswerten aus der Vergangenheit, in einem langwierigen, aufwendigen, kostenintensiven und hochpolitisierten Verfahren erteilt wird. Dabei setzt die Genehmigung (auch von Feldversuchen) voraus, dass Informationen über Identifizierungs-, Nachweis- und Überwachungsverfahren vorgelegt werden. Bislang existiert aber keine Methode, mit welcher im Fall von bloßen Mutationen nachgewiesen werden könnte, ob die Mutationen überhaupt technisch und, wenn ja, mit welcher Technik sie erzeugt wurden (ZKBS, 2019). Dieses Problem wirkt sich auch auf das Koexistenzregime aus, ${ }^{27}$ z. B. bei Fragen der Beweisführung in Haftungsfällen wegen „gentechnischer Kontamination“ konventioneller oder ökologischer Produkte. Außerdem können die EU-Mitgliedstaaten den Anbau genomeditierter Pflanzen durch Inanspruchnahme der Opt-out-Regeln z. B. aus bestimmten sozioökonomischen Gründen beschränken oder ganz verbieten. Schließlich müssen die Vorschriften über die gentechnikspezifische Kennzeichnung und Rückverfolgbarkeit auch für Produkte, die genomeditierte Organismen enthalten, daraus bestehen oder aus solchen gewonnen wurden, eingehalten werden.

26 Ferner dürfen auch im ökologischen Landbau keine genomeditierten Organismen verwendet werden (vgl. Leopoldina et al., 2019: 19).

27 Das eigentlich darauf angelegt ist, die Wahlfreiheit der Landwirte und Verbraucher zu sichern und deshalb ein störungsfreies Nebeneinander von gentechnischen, konventionellen und ökologischen Produkten bzw. Produktions- und Vermarktungsketten zu gewährleisten. 


\subsubsection{Folgen für das „geschlossene System“}

Für die Verwendung von GVO im geschlossenen System (z. B. in Laboren, Gewächshäusern, Tierhaltungsräumen, Industrieanlagen) gilt auf Unionsebene ein eigenes, von den vorstehend genannten Regeln separiertes, Regelwerk, nämlich die Richtlinie 2009/41/ $E G,{ }^{28}$ die ihren - auf gentechnisch veränderte Mikroorganismen (GVM) beschränkten - Anwendungsbereich autonom definiert. Deshalb erstreckt sich das Urteil des EuGH, das sich ausschließlich auf die Auslegung der Richtlinie 2001/18/EG bezieht und dabei vollständig im Kontext dieser Richtlinie verbleibt, nicht automatisch auf die GVM-Definition und die Ausnahmebestimmungen der Richtlinie 2009/41/EG.

Allerdings sind die GVO- bzw. GVM-Definitionen sowie die Ausnahmebestimmungen der beiden Richtlinien 2001/18/EG und 2009/41/EG nach Wortlaut und Regelungsstruktur praktisch identisch. Identisch sind auch die Schutzzwecke beider Richtlinien. Dass die Richtlinie 2009/41/EG in diesem Zusammenhang das Vorsorgeprinzip (anders als die Richtlinie 2001/18/EG) nicht ausdrücklich erwähnt, ist dabei unproblematisch. Denn dessen Bedeutung für die Auslegung und Anwendung der Richtlinie 2009/41/ EG ergibt sich unmittelbar aus dem primären Unionsrecht (Art. 191 Abs. 2 Unterabs. 1 Satz 2 AEUV). Schließlich werden beide Richtlinien von dem gleichen Gedanken beherrscht, wonach sie nur für solche GVO bzw. GVM keine Geltung haben sollen, die sich als empirisch sicher erwiesen haben. ${ }^{29}$ Daher sprechen die besseren Gründe dafür, dass genomeditierte Mikroorganismen im Lichte des EuGH-Urteils als GVM von der Richtlinie 2009/41/EG erfasst und ausnahmslos reguliert werden (Kahrmann/Leggewie, 2018: 764; Dederer, 2019: 243 f.; Spranger, 2019a; Dederer, 2020: 51 f.; Voigt, 2021: 51 ff.).

\subsubsection{Welthandelsrechtliche Folgen}

Die gentechnikrechtliche Marktzulassungspflicht für den Import von GVO in die EU wirkt sich welthandelsrechtlich als Handelshemmnis für Drittstaaten (z. B. Argentinien, Kanada oder die USA) aus. Dessen Rechtfertigung könnte daran scheitern, dass die Union für Organismen, welche aus herkömmlicher Zufallsmutagenese hervorgegangen sind, keine Zulassungspflicht vorgesehen hat, obwohl jene Methoden in weit größerem Maße Mutationen und Off-Target-Effekte und damit potenzielle, nicht vorab umfassend bewertete Risiken für Umwelt und Gesundheit hervorrufen als Techniken

28 Richtlinie 2009/41/EG des Europäischen Parlaments und des Rates vom 6. Mai 2009 über die Anwendung genetisch veränderter Mikroorganismen in geschlossenen Systemen. In: ABl. EU L 125, 21.05.2009: 75-97.

29 Vgl. Erwägungsgrund 17 der Richtlinie 2001/18/EG und Erwägungsgrund 25, Art. 3 Abs. 1 lit. b, Anhang II Teil B Richtlinie 2009/41/EG. 
der Genomeditierung, also der gezielten Mutagenese (Leopoldina et al., 2019: 10/14/32; zu gesellschaftlichen Einstellungen gegenüber Mutagenese und Genome-Editing siehe Hampel et al., Kap. 24). Deshalb lässt sich argumentieren, dass eine Zulassungspflicht für genomeditierte Mutagenese-Organismen nicht notwendig und dass ein unterschiedliches Schutzniveau für konventionelle Mutagenese-Organismen einerseits und genomeditierte Organismen andererseits diskriminierend, willkürlich und deshalb inkonsistent, also mit dem Recht der Welthandelsorganisation (World Trade Organization - WTO) unvereinbar ist (Leopoldina et al., 2019: 27-29; anders Spranger, 2019b: 11-14).

\subsubsection{Wirtschaftliche Folgen}

Die beschriebenen rechtlichen Folgen ziehen weitreichende wirtschaftliche Folgen nach sich, namentlich für Saatgutentwickler bzw. Sortenzüchter und für Tierzüchter. Der Aufwand für Forschung und Entwicklung bis zur Marktzulassung wird sich, wegen der Anforderungen vor allem der Genehmigungsverfahren für Freilandversuche und Vermarktung, deutlich erhöhen (vgl. McFadden/Smyth, 2019: 451; Qaim, 2020: 144). Die Rückverfolgbarkeitspflichten lassen die Kosten im Verlauf der Produktions- und Vertriebskette gleichfalls steigen. Dabei ist nicht gesichert, dass sich alle diese Kosten im Zuge des Produktabsatzes vollständig amortisieren (Purnhagen/Wesseler, 2020: 6-9). Denn bspw. die gentechnikspezifische Kennzeichnung kommt einem faktischen, wenn auch vom Recht formaliter nicht intendierten, Warnhinweis an die Abnehmer gleich und hindert deshalb nach aller bisherigen Erfahrung die Verkäuflichkeit des betreffenden Produkts. Opt-out-Regeln können den Anbau genomeditierter Pflanzen vollständig ausschließen und damit den Kauf bzw. Verkauf des betreffenden Saatguts gänzlich unattraktiv machen. In gleicher Weise kann sich ein nationales Koexistenzregime, zumal wenn es eine verschuldensabhängige Haftung wegen „GVO-Verunreinigung“ vorsieht, prohibitiv auswirken.

Die Nutzung der Genomeditierung dürfte daher nicht nur für Saatgutentwickler bzw. Sortenzüchter und für Tierzüchter abschreckend sein (Leopoldina et al., 2019: 21), sondern auch für Exporteure in die EU (vgl. Gocht et al., 2020: 21; Smyth/Lassoued, 2019: 338). Daraus können sich gewaltige, nicht nur die Landwirtschaft, sondern auch die Umwelt erfassende, Rückkopplungseffekte für Drittstaaten, insbesondere Entwicklungsländer (Leopoldina et al., 2019: 29), aber auch für die EU selbst ergeben (Gocht et al., 2020: 15-20). Mit nachteiligen Auswirkungen ist insbesondere für die Wettbewerbsfähigkeit der Landwirtschaft und deren Attraktivität für in- und ausländische Investoren zu rechnen (Smyth/Lassoued, 2019: 338-339). Zugleich wird durch diese Entwick- 
lungen aber auch der europäische Forschungsstandort massiv geschwächt (Leopoldina et al., 2019: 19-21; Smyth/Lassoued, 2019: 338; siehe auch van den Daele/Broer, Kap. 21).

\subsection{Schlussfolgerungen}

Die rechtlichen und wirtschaftlichen Folgen des EuGH-Urteils lassen eine schnelle Änderung der Rechtslage unumgänglich erscheinen. Vorschläge hierzu liegen weitgehend ausformuliert auf dem Tisch (Leopoldina et al., 2019: 33-34; siehe für einen differenzierten, Aspekte der Nachhaltigkeit in den Blick nehmenden Vorschlag Renn, Kap. 23). Es verhält sich dabei auch keineswegs so, als bräuchte der EU-Gesetzgeber stets unabsehbar viele Jahre für eine Rechtsänderung. ${ }^{30}$ Der Unionsgesetzgeber mag wegen der fraglichen Akzeptanz der Gentechnik in der Bevölkerung eine Änderung der Richtlinie 2001/18/EG ungern in Angriff nehmen zu einer aktuellen Erhebung der Einstellung in der deutschen Bevölkerung siehe Hampel et al., Kap. 24). Hier ist aber politische Führung gefragt in dem Sinne, dass die Politik, sofern sie die Einsicht in die Notwendigkeit einer Deregulierung selbst gewonnen hat, dieser Einsicht gemäß handelt und den sich daraus ergebenden Weg der Rechtsänderung mit dem Willen zur Überzeugung gegenüber der Öffentlichkeit klar kommuniziert und vorzeichnet.

\subsection{Literaturverzeichnis}

Beck, F. (2019): All about that risk? A (re-)assessment of the CJEU's reasoning in the „genome editing“ case. In: Zeitschrift für Europäisches Umwelt- und Planungsrecht (EurUP) 17(2): 246-255.

Dederer, H.-G. (2019): Genomeditierung ist Gentechnik. Eine kritische Analyse des EuGH-Urteils Confédération paysanne u.a. In: Zeitschrift für Europäisches Umwelt- und Planungsrecht (EurUP) 17(2): 236-245.

Dederer, H.-G. (2020): Das EuGH-Urteil vom 25.07.2018 und dessen Folgen für die Rechtslage nach dem Gentechnikrecht. In: Bayerisches Landesamt für Gesundheit und Lebensmittelsicherheit (Hrsg.): 8. Fachtagung Gentechnik. Erlangen: 41-52.

Faltus, T. (2018): Mutagene(se) des Gentechnikrechts. Das Mutagenese-Urteil des EuGH schwächt die rechtssichere Anwendung der Gentechnik. In: Zeitschrift für Umweltrecht (ZUR) 10: 524-534.

30 In Notsituationen wie der COVID-19-Pandemie vermag der Gesetzgeber sogar binnen weniger Wochen zu handeln. Siehe die innerhalb von weniger als einem Monat angenommene Verordnung (EU) 2020/1043 des Europäischen Parlaments und des Rates vom 15. Juli 2020 über die Durchführung klinischer Prüfungen mit genetisch veränderte Organismen enthaltenden oder aus solchen bestehenden Humanarzneimitteln zur Behandlung oder Verhütung der Coronavirus-Erkrankung (COVID-19) und deren Abgabe. In: ABl. L 231 vom 17.07.2020: 12-16. 
Gocht, A. et al. (2020): Economic and environmental consequences of the ECJ genome editing judgement in agriculture. Thünen Working Paper, No. 150. Johann Heinrich von Thünen-Institut, Braunschweig.

Gürtler, P. et al. (2019): Genome Editing. In: Bayerisches Landesamt für Gesundheit und Lebensmittelsicherheit (Hrsg.): Band 11 der Schriftenreihe Gentechnik für Umwelt- und Verbraucherschutz. Erlangen.

Kahrmann, J./Leggewie, G. (2018): Gentechnikrechtliches Grundsatzurteil des EuGH und die Folgefragen für das deutsche Recht. In: Natur und Recht (NuR) 40: 761-765.

Leopoldina Nationale Akademie der Wissenschaften/Deutsche Forschungsgemeinschaft/Union der deutschen Akademien der Wissenschaften (2019): Wege zu einer wissenschaftlich begründeten, differenzierten Regulierung genomeditierter Pflanzen in der EU. Halle (Saale).

McFadden, B. R./Smyth, S. J. (2019): Perceptions of genetically engineered technology in developed areas. In: Trends Biotechnol. 37(5): 447-451.

Purnhagen, K./Wesseler, J. (2020): EU regulation of new plant breeding technologies and their possible economic implications for the EU and beyond. In: Applied Economic Perspectives and Policy: 1-17. DOI: 101002/aepp.13084.

Qaim, M. (2020): Role of new plant breeding technologies for food security and sustainable agricultural development. In: Applied Economic Perspectives and Policy 42(2): 129-150.

Seitz, C. (2018): Modifiziert oder nicht? Regulatorische Rechtsfragen zur Genoptimierung durch neue biotechnologische Verfahren. In: Zeitschrift für Europäisches Wirtschaftsrecht (EuZW) 18: 757-764.

Smyth, S. J./Lassoued, R. (2019): Agriculture R\&D implications of the CJEU's gene-specific mutagenesis ruling. In: Trends Biotechnol. 37(4): 337-340.

Spranger, T. M. (2018): Neue Techniken und Europäisches Gentechnikrecht. In: Neue Juristische Wochenschrift (NJW): 2929-2930.

Spranger, T. M. (2019a): Memorandum zur Frage der Übertragbarkeit der Ausführungen des Europäischen Gerichtshofes in der Rs. C-528/16 auf den Regulierungsbereich der Systemrichtlinie 2009/41/EG. Unter: https://www.bfn.de/fileadmin/BfN/recht/Dokumente/System_Memorandum_ final.pdf [21.01.2021].

Spranger, T. M. (2019b): Memorandum zur Frage der welthandelsrechtlichen Implikationen des Urteils des Europäischen Gerichtshofes in der Rs. C-528/16. Unter: https://www.bfn.de/fileadmin/ BfN/recht/Dokumente/Welthandelsrecht_Memorandum_final_2.pdf [21.01.2021].

van der Meer, P. et al. (2021): The status under EU law of organisms developed through novel genomic techniques. In: European Journal of Risk Regulation: 1-20. DOI:10.1017/err.2020.105.

Voigt, B. (2018): Anmerkung: Genomeditierung = Gentechnik. In: Zeitschrift für das gesamte Lebensmittelrecht (ZLR) 05: 654-663. 
Voigt, B. (2021): GVO oder nicht? Eine Untersuchung zur Auslegung von GVO-Definitionen im Unionsund Völkerrecht mit Blick auf neue molekularbiologische Züchtungstechniken. LIT Verlag, Münster.

Voigt, B./Münichsdorfer, A. (2019): Regulation of genome editing in plant biotechnology: European Union. In: Dederer, H.-G./Hamburger, D. (Hrsg.): Regulation of genome editing in plant biotechnology. A comparative analysis of regulatory frameworks of selected countries and the EU. Springer, Cham: $137-238$.

Wasmer, M. (2019): Roads forward for european GMO policy - Uncertainties in wake of ECJ judgment have to be mitigated by regulatory reform. In: Front. Bioeng. and Biotechnol. 7: 132. DOI:10.3389/ fbioe.2019.00132.

ZKBS = Zentrale Kommission für die Biologische Sicherheit (2019): Zur Identifizierbarkeit von Genomeditierungen in Pflanzen. Kommentar zu Y. Bertheau. Unter: https://www.bvl.bund.de/ ZKBS/DE/Kommentare/02_IdentGenomedit_Pflanzen/IdentGenomedit_Pflanzen_node.html [21.01.2021]. 